Stefanny Christie Monteiro Titon

Variações na resposta imune, níveis plasmáticos de corticosterona e testosterona e condição corpórea de sapos Brasileiros em resposta ao cativeiro

Immune response, plasma corticosterone and testosterone levels, and body condition variation of Brazilian toads in response to captivity

São Paulo 
Stefanny Christie Monteiro Titon

Variações na resposta imune, níveis plasmáticos de corticosterona e testosterona e condição corpórea de sapos Brasileiros em resposta ao cativeiro

Immune response, plasma corticosterone and testosterone levels, and body condition variation of Brazilian toads in response to captivity

Tese apresentada ao Instituto de Biociências da Universidade de São Paulo, para a obtenção de Título de Doutor em Ciências, na Área de Fisiologia Geral.

Orientador: Fernando Ribeiro Gomes Co-orientador: Pedro A.C.M. Fernandes

São Paulo 
Titon, Stefanny Christie Monteiro

Variações na resposta imune, níveis plasmáticos de corticosterona e testosterona e condição corpórea de sapos Brasileiros em resposta ao cativeiro / Stefanny Christie Monteiro Titon; orientador Fernando Ribeiro Gomes. -São Paulo, 2017.

\section{$134 \mathrm{f}$.}

Tese (Doutorado) - Instituto de Biociências da Universidade de São Paulo, Departamento de Fisiologia.

1. estresse. 2. corticosterona. 3. testosterona. 4. resposta imune. 5. condição corpórea. I. Gomes, Fernando Ribeiro, oriente. II. Título.

\section{Comissão Julgadora}

Prof(a). Dr(a).

Prof(a). Dr(a).
Prof(a). Dr(a).

Prof(a). Dr(a).

Prof(a). Dr(a). 


\section{DEDICATÓRIA}

Dedico este trabalho:

A Deus, meu guia e sustento, por toda a estrada.

Aos meus pais Luiz e Lúcia, que são a minha base, me apoiando e me incentivando neste sonho em todo o tempo, e meus irmãos Rafael e Renata sempre presentes nesta caminhada.

Ao meu esposo Braz Jr. meu meu amor, meu porto seguro, meu lar.

Aos meus sogros Cida e Braz, que com muito zelo me abraçaram em sua família, e me apoiam continuamente. 


\section{AGRADECIMENTOS}

Ao meu orientador Dr. Fernando Ribeiro Gomes, que sempre acreditou no meu potencial, me ajudou com todas as dúvidas e inseguranças do projeto desenvolvido, que me apoiou de maneira expetacular nesta porção, meu doutorado, da caminhada de pesquisa interminável.

Ao meu co-orientador: Pedro Augusto Carlos Magno Fernandes, que com uma contribuição inestimável me ajudou a direcionar o meu trabalho por caminhos maravilhosos.

Ao meu esposo, Braz Titon Junior, meu parceiro de coletas, experimentos, análises estatísticas e muitas discussões, além de tanta dedicação e compreensão a todo o meu trabalho. Ter ele ao meu lado em todas as etapas sempre foi reconfortante.

A minha amiga que São Paulo me trouxe de presente para a vida Vânia Regina de Assis, que esteve ao meu lado sempre, tanto na elaboração, execução de experimentos e escrita dos textos, mas também me dando apoio emocional, que é algo muito, mas muito valioso. 
Aos amigos e colaboradores Adriana Maria Giorgi Barsotti, Bruna de

Oliveira Cassettari e Gabriela Kinker, pela amizade e ajuda em momentos preciosos. Assim como a Nicolle G. T. Q. Hazarbassanov e Eduardo B. Fernandes, técnicos de laboratório, por toda a ajuda.

A todos os colegas do laboratório de Comportamento e Fisiologia Evolutiva pelas conversas e amizade construída no ambiente de trabalho.

A professora Cristina O. M. S. Gomes pela assistência com relação a questões metodológicas e colaborações, o que me ajudaram a realizar este trabalho de uma maneira singular.

A todos do Departamento de Fisiologia Geral, especialmente a Roseli Silva Santos e Erika H. Takamoto de Camargo por trabalho desenvolvido, a ajuda de vocês foi essencial nesta caminhada.

Ao ajudante de campo Marcos D. Franco, presente nas noites de coleta na cidade de Luiz Antônio.

Com carinho...

Stefanny Christie Monteiro Titon 
Este trabalho foi financiado por:

Fundação de Amparo à Pesquisa do Estado de São Paulo (FAPESP):

Projeto de Auxílio concedido à FRG (2013/00900-1)

Projeto Temático concedido à CNI (2014/16320-7)

Conselho Nacional de Desenvolvimento Cientifico e Tecnológico (CNPq):

Bolsa de doutorado concedida a SCMT (142455/2013-0 GD)

Programa de Pós-graduação em Fisiologia Geral - Departamento de Fisiologia - Instituto de Biociências - USP - São Paulo.

Departamento de Fisiologia - Instituto de Biociências - USP - São Paulo. 


\section{SUMÁRIO}

RESUMO 9

GENERAL

INTRODUÇÃOO

Relações entre estresse resposta imune e andrógenos 13

Imunomodulação mediada por glicocorticóides $\quad 15$

Imunomodulação mediada por andrógenos 17

$\begin{array}{ll}\text { Justificativa } & 19\end{array}$

CONCLUSÕES

REFERÊNCIAS 


\section{RESUMO}

O aumento dos níveis plasmáticos de glicocorticoides com concomitante diminuição de níveis plasmáticos de andrógenos em resposta a estressores é comumente observado em diversos vertebrados. Adicionalmente, os glicocorticoides, bem como os andrógenos, exercem importantes efeitos imunomodulatórios. Populações naturais de anfíbios respondem a agentes estressores de maneiras variadas, incluindo aumento dos níveis plasmáticos de corticosterona (principal glicocorticoide em anfíbios - CORT), alterações na resposta imune e a diminuição dos níveis plasmáticos de testosterona (T). Utilizando o cativeiro como um agente estressor para populações naturais de anfíbios, investigamos os efeitos da manutenção em cativeiro sobre os níveis plasmáticos de CORT e T, resposta imune (capacidade bactericida do plasma - CBP e fagocitose de células peritoneais) e condição corpórea de indivíduos machos de Rhinella schneideri e $R$. icterica, a partir de indivíduos coletados em populações naturais e mantidos em cativeiro por 60 e 90 dias, respectivamente. Machos adultos foram amostrados em campo, para coleta de dados basais, transportados e mantidos em cativeiro, onde amostras foram coletadas após 7, 30, 60 e 90 dias. Inicialmente, foi realizada a padronização da metodologia do ensaio de fagocitose das células peritoneais em sapos. Nossos resultados demonstraram que a o ensaio de fagocitose e a análise por citometria de fluxo por imagem resultam em um método confiável e preciso para a análise de atividade fagocítica de células peritoneais de sapos. Em resposta ao cativeiro, ambas as espécies ( $R$. schneideri e $R$. icterica) apresentaram aumento dos níveis plasmáticos de CORT, diminuição dos níveis plasmáticos de $\mathrm{T}$ e resposta imune (CBP e fagocitose), indicando uma condição de estresse crônico. Indivíduos da espécie $R$. schneideri apresentaram uma acentuada perda da condição corpórea em resposta ao cativeiro, enquanto os indivíduos da espécie $R$. icterica mantiveram a condição corpórea ao longo do tempo em cativeiro. A CBP esteve positivamente correlacionada com a CORT plasmática quando não houve variação da condição corpórea ( $R$. icterica), enquanto que em $R$. schneideri, os 
indivíduos que perderam massa ao longo do tempo apresentaram níveis elevados de CORT plasmática e menores valores de CBP. Adicionalmente, os níveis plasmáticos de T estavam positivamente correlacionados com a fagocitose em ambas as espécies estudadas. Estes resultados indicam que a manutenção em cativeiro pode ser considerada um estressor crônico para sapos, uma vez que promove o aumento de CORT e diminuição de $\mathrm{T}$ e resposta imune nestes animais. De maneira associada, a variação da resposta imune em resposta à manutenção em cativeiro depende do parâmetro avaliado e tempo de resposta (dias em cativeiro). Nossos resultados ainda sugerem que a imunomodulação nas condições de cativeiro em sapos pode estar associada às variações de CORT e T plasmática, dependendo de sinalização da condição corpórea do animal.

Palavras-chave: Estresse, corticosterona, testosterona, resposta imune, condição corpórea. 


\section{ABSTRACT}

Increased plasma glucocorticoid levels with concomitant decrease in plasma androgen levels in response to stressors are commonly observed in several vertebrates. In addition, glucocorticoids, as well as androgens, plays important immunomodulatory effects. Natural populations of amphibians respond to stressors in different ways, including increased corticosterone (main glucocorticoid in amphibians - CORT) plasma levels, changes in immune responses, and decreased plasma testosterone (T) levels. Given that captivity is a stressor for amphibian natural populations, we investigated the effects of captivity maintenance on plasma CORT and T levels, immune response (plasma bacterial killing ability - BKA and phagocytosis of peritoneal cells) and body condition of male individuals of Rhinella schneideri and $R$. icterica, from individuals collected in natural populations and kept in captivity for 60 and 90 days, respectively. Adult males were sampled in the field for baseline measurements, transported and kept in captivity, where samples were collected after 7, 30, 60 and 90 days. Initially, we standardized the phagocytosis of peritoneal cells assay for toads. Our results demonstrated that the phagocytosis assay and the imaging flow cytometry analysis result in a reliable and accurate method for the analysis of phagocytic activity of peritoneal cells of toads. In response to captivity, both species ( $R$. schneideri and $R$. icterica) showed increased plasma CORT, decreased plasma T levels and immune response (BKA and phagocytosis), indicating a chronic stress condition. Individuals of $R$. schneideri showed accentuated body condition loss in response to captivity, while individuals of $R$. icterica displayed sustained body condition over time in captivity. BKA was positively correlated with plasma CORT when there was no change in body condition ( $R$. icterica), whereas in $R$. schneideri, individuals showing the highest body mass loss over time, also presented the highest CORT levels and the lowest BKA values. Additionally, plasma T levels were positively correlated with phagocytosis in both studied species. These results indicate that captivity maintenance can be considered a chronic 
stressor for toads, promoting increased CORT and decreased $\mathrm{T}$ and immune response in these animals. Moreover, the variation in immunity in response to captivity maintenance depends on the evaluated parameter and time (days in captivity). Our results further suggest that immunomodulation in captivity conditions in toads may be associated with changes in plasma CORT and T, depending on the individual's body condition.

Keywords: Stress, corticosterone, testosterone, immune response, body condition. 


\section{INTRODUÇÃO}

\section{Relações entre estresse, resposta imune e andrógenos}

Em resposta a um estímulo estressor, ocorre ativação do sistema nervoso central e concomitante ativação do eixo hipotálamo-hipófise-adrenais/interrenais (HPA/I) (Sapolsky et al., 2000; Sapolsky, 2002). Esta ativação simultânea do sistema nervoso e endócrino resulta na liberação de catecolaminas e glicocorticoides (GC), os quais promovem alterações fisiológicas como: aumento do tônus muscular, aumento da disponibilização de substratos energéticos e imunomodulação (Sapolsky, 2000). Embora a resposta de estresse apresente efeitos benéficos, que promovem a sobrevivência do animal a uma determinada condição estressante, a ativação frequente do sistema nervoso e do eixo HPA/I em resposta a estressores pode resultar em efeitos deletérios para o animal, como a inibição da reprodução e supressão da resposta imune (Sapolsky et al., 2000; Sapolsky, 2002; Marketon e Glaser, 2008).

Diversos estudos mostram que a inibição de diferentes pontos do eixo hipotálamohipófise-gônadas em resposta a ativação do eixo HPA/I tem sido demonstrada em vertebrados, incluindo anfíbios (Sapolsky, 2002; Deviche et al, 2010; Tokarz e Summers, 2011; Barsotti et al., 2017). Como resultado, frequentemente observamos a inibição do comportamento reprodutivo, assim como a diminuição dos níveis plasmáticos de andrógenos em resposta a diferentes estressores em machos (Paolucci et al., 1990; Moore et al., 1991; Sapolsky et al., 2000; Jones e Bell, 2004; Deviche et al., 2010; Narayan et al., 2012; Assis, 2015). Os andrógenos, por sua vez, além de exercerem um importante papel na reprodução, também apresentam ações imunomoduladoras (Nava-Castro et al., 2012). As células imunes, uma vez que apresentam receptores para andrógenos, podem ser moduladas diretamente pelos andrógenos (Nava-Castro et al., 2012). É importante ressaltar que ações indiretas dos andrógenos também podem ser observadas, uma vez que a testosterona ( $\mathrm{T}$ - principal andrógeno nos machos) pode competir pelas globulinas de ligação de GCs, alterando a fração livre destes 
hormônios (Bradley et al., 1980; McDonald et al., 1981; Bradley, 1987 McEwen et al., 1997). Portanto, uma vez que as células imunes também apresentam receptores para GCs, estando sujeitas a modulação direta dos GCs (Wiegers e Reul, 1998), é possível que os andrógenos, associados aos GCs, exerçam um papel fundamental na regulação da resposta imune, particularmente durante a resposta de estresse.

Em relação ao sistema imune, a resposta de estresse promove uma imunomodulação direta, mediada pela ativação do sistema nervoso central (inervação direta dos órgãos linfoides), e também, via ação hormonal, através, principalmente, da atuação das catecolaminas e dos GCs sobre as células imunes (Marketon e Glaser, 2008). Em resposta ao estresse agudo, o sistema imune apresenta alterações como redistribuição de leucócitos (linfócitos T e B, células naturalkiller, e monócitos) do sangue para tecidos periféricos e linfonodos, resultando em uma diminuição do número de células imune na corrente sanguínea (Dhabhar, 2009; Dhabhar, 2014). De maneira associada, durante uma resposta de estresse agudo, ocorre um aumento da resposta inflamatória, assim como de diversos componentes da resposta inata e adquirida (Dhabhar, 1999; Barriga et al., 2001; Dhabhar, 2009; Graham et al., 2012). Por outro lado, o estresse crônico apresenta, em sua maioria, efeitos imunossupressores. De fato, diminuições da resposta inflamatória e reduções na atividade de células fagocitárias e de componentes da resposta humoral e mediada por células são comumente observadas em resposta ao estresse crônico (Hoffman-Goetz et al, 1986; Yin et al, 1995; Dhabhar, 2009; Dhabhar, 2014; Assis et al., 2015; Neuman,-Lee et al., 2015). Deste modo, apesar da imunomodulação associada à resposta de estresse ser muito mais refinada do que o exposto (Dhabhar, 2014), observamos que os efeitos estimulatórios ou supressores da resposta imune apresentam uma forte relação com a duração da resposta de estresse.

Variações na amplitude e duração da elevação dos níveis plasmáticos de corticosterona (CORT - principal GC em anfíbios), assim como nas alterações no perfil da resposta imune, 
têm sido reportadas em diversos anfíbios expostos a diferentes agentes estressores. Algumas espécies de anuros Brasileiros tem demonstrado um aumento da CORT e diminuição da capacidade bactericida plasmática em resposta ao estresse de contenção (Gomes et al., 2012) e cativeiro (Assis et al., 2015). Entretanto, apesar de Graham et al. (2012) terem demonstrado um efeito imunossupressor sobre a capacidade de lise do plasma, também foi observado um efeito estimulador sobre o burst oxidativo de leucócitos sanguíneos em resposta ao estresse de restrição em sapos Australianos. Por outro lado, Assis (2015) demonstrou que algumas espécies de anuros não apresentam variação da capacidade bactericida plasmática mesmo quando submetidos ao estresse de contenção de movimentos por 24h. Estes estudos demonstram que diferentes segmentos do sistema imune podem ser modulados de forma distinta em resposta a estressores em diversos anfíbios. De maneira associada, uma diminuição nos níveis de T tem sido frequentemente observada em resposta a estressores em anuros (Jones e Bell, 2004; Narayan et al., 2012; Assis, 2015). Entretanto, estudos que incorporem medidas de CORT, T e reposta imune com anuros são escassos (Assis, 2015), principalmente no contexto de estresse crônico.

\section{Imunomodulação mediada por glicocorticóides}

Os GCs são hormônios esteroides que promovem alterações fisiológicas que garantem a sobrevivência do animal durante a resposta de estresse (Sapolsky et al., 2000). Estudos realizados com diversos vertebrados demonstraram que elevações agudas de GCs promovem um aumento em diversos aspectos da resposta imune: resposta mediada por células (Dhabhar e McEwen, 1999); fagocitose de leucócitos do sangue (Graham et al., 2012; Assis et al., 2017); aglutinação mediada por anticorpos (McCormick e Langkilde, 2014); os quais poderiam estar associados a uma maior resposta imune em um período de recuperação pós estresse (Dhabhar, 2014). De fato, GCs liberados de forma aguda na corrente sanguínea promovem a liberação de 
moléculas constituintes da resposta imune humoral que podem, por sua vez, ativar os mediadores celulares da resposta imune e direcionar os neutrófilos e linfócitos circulantes para tecidos específicos (Wiegers \& Reul, 1998; Riccardi, et al, 2002; Cavalcanti et. al., 2006). Adicionalmente, estudos in vitro demonstraram que níveis fisiológicos de GCs aumentam a fagocitose e a quimiotaxia em macrófagos peritoneais de ratos (Barriga et al., 2002; Zhong et al., 2013) e a proliferação celular em ovelhas (Ciliberti et al., 2016), evidenciando o efeito imuno estimulador do aumento agudo dos GCs.

Por outro lado, níveis cronicamente elevados dos GCs apresentam efeitos majoritariamente imunossupressores (Dhabhar, 2014). Estudos abordando elevações crônicas experimentais de GCs demonstraram que estes hormônios podem retardar o processo de cicatrização, diminuir a resposta imune mediada por células, bloquear e inibir a maturação e desenvolvimento dos linfócitos, além de atuarem na diminuição da massa e função dos órgãos linfoides (Ahmed et. al., 1985; Wiegers \& Reul, 1998; Dhabhar e McEwen, 1999; Riccardi, et al, 2002; Cavalcanti et. al., 2006; Thomas e Woodley, 2015), caracterizando a função imunossupressora destes esteroides. Tratamentos in vitro, demonstraram que o uso crônico de GCs, assim como altas doses destes hormônios também têm apresentam efeitos supressores sobre a resposta imune celular, atuando na diminuição da proliferação de células do sangue e da atividade (quimiotaxia e fagocitose) de macrófagos (Wiegers et al., 1993; Lim et al., 2007; Ciliberti et al., 2016). Adicionalmente, estudos realizados com camundongos demonstraram que baixas doses de CORT aumentam a produção de citocinas (TNF e IL-6), enquanto que altas doses do mesmo hormônio apresentam um efeito oposto (Chantong et al., 2012), especialmente quando combinada com uma injeção de lipopolissacarídeos (Liao et al., 1995). Por outro lado, é interessante ressaltar que a mesma dose de GCs pode apresentar tanto efeito supressor quanto estimulador sobre a resposta imune, variando de acordo com o tempo de exposição das células a estes hormônios (Wiegers et al., 1995; Ciliberti et al., 2016). Deste modo, os efeitos bimodais 
dos GCs parecem estar associados a variação na duração e intensidade da resposta de estresse, além de também estar relacionados a ação dos distintos receptores de GCs (Wiegers et al., 1993; Wiegers \& Reul, 1998; Salpolsky et al., 2000; Chantong et al., 2012).

Embora os efeitos imunomodulatórios diretos dos GCs estejam bem estabelecidos, sabese que a condição corpórea do indivíduo parece ser fundamental durante a modulação da resposta imune mediada pelo estresse. Em condições de vida livre, iguanas com maiores níveis de CORT apresentaram uma menor condição corpórea e resposta inflamatória (Berger et al., 2005). De maneira semelhante, trabalhos com mamíferos e aves demonstraram que, em resposta ao estresse agudo, o aumento da resposta imune concomitante ao aumento dos níveis circulantes de GCs, normalmente ocorre quando o indivíduo apresenta uma boa condição corpórea (Dhabhar, 2009; Harms et al., 2010). Por outro lado, Bourgeon et al. (2007) demonstraram que patos submetidos a um desafio com LPS apresentaram uma correlação positiva entre a condição corpórea e a resposta imune (níveis plasmáticos de oxido nítrico), entretanto, os animais com maior resposta imune e condição corpórea foram os que apresentaram os menores níveis de CORT. Estes estudos demonstram que os GCs associados com a sinalização de condição corpórea parecem exercer um papel fundamental sobre a resposta imune em vertebrados.

\section{Imunomodulação mediada por andrógenos}

Apesar de exercerem um papel intimamente relacionado à reprodução, os hormônios sexuais, incluindo a testosterona ( $\mathrm{T}$ - principal andrógeno em vertebrados), são importantes reguladores de diversos aspectos da resposta imune inata e adquirida nos vertebrados (Ahmed et al., 2010). Os efeitos imunossupressores da T são bastante conhecidos, e incluem a redução de tecidos linfoides, assim como a diminuição da resposta imune celular e humoral (Grossman, 1985; Casto et al, 2001; Ahmed et al., 2010; Nava-Castro et al, 2012). Estudos in vivo e in vitro demonstraram que o tratamento com a $\mathrm{T}$ diminui a proliferação de linfócitos, produção de 
anticorpos, assim como a atividade celular de macrófagos, representada pela fagocitose, produção de nitrito e interleucina-1 em diversos táxons (Savita e Rai, 1998; Casto et al., 2001; Mondal e Rai, 2002; Tripathi e Singh, 2014). Adicionalmente, aplicações agudas e sustentadas de $\mathrm{T}$ podem reduzir a atividade de células natural-killer, assim como diminuir as respostas humoral e mediada por células (Inman, 1978; Grossman, 1984; Ahmed et al., 1985; Hou e Zheng, 1988). Entretanto, embora uma imunossupressão associada a T em animais seja relatada para diversas espécies de vertebrados, uma meta-análise incluindo mamíferos, aves e répteis demonstrou que o efeito supressor da T varia de acordo com o tipo de resposta imune estudado, bem como dentre os grupos filogenéticos (Roberts et al., 2004).

Efeitos estimuladores da $\mathrm{T}$ são observados, principalmente, relacionados à resposta inflamatória (McCrohon et al., 1999; Greenman et al., 2005; Martin et al., 2008; Gilliver, 2010). Entretanto, há estudos demonstrando que a T também influencia positivamente a atividade celular de macrófagos (aumento da produção de óxido nítrico) e a produção de anticorpos em alguns animais (Chao et al., 1994; Evans et al., 2000; Peters, 2000). Adicionalmente, estudos recentes têm demonstrado que os efeitos estimuladores da T são observados, principalmente, associados a uma boa condição corpórea do animal. Desprat et al. (2015) demonstraram que pererecas tratadas com T, que apresentaram maiores níveis plasmáticos de T e com uma melhor condição corpórea, também apresentaram uma maior resposta inflamatória quando comparados com indivíduos com menor condição corpórea e com o grupo placebo (sem aplicação de T). Por outro lado, Roberts et al. (2007) demonstraram que aves tratadas com implantes de T, demonstrando menor massa corpórea, mas apresentando maiores níveis de $\mathrm{T}$ concomitantemente com maiores níveis de CORT, exibiram uma maior produção de anticorpos em resposta a um dado antígeno, sugerindo uma possível interação entre estes dois esteroides na regulação do sistema imune (Roberts et al., 2007). Consequentemente, é possível que a T possa participar de maneira fundamental da regulação da resposta imune durante a resposta de 
estresse; e que a interação entre a CORT, T e condição corpórea sejam determinantes no controle do sistema imune em resposta a estressores.

\section{Justificativa}

Diversos estudos abordam diferentes respostas fisiológicas associadas à resposta de estresse do animal (Sapolsky et al., 2000), no entanto, o enfoque se dá em poucos aspectos fisiológicos em conjunto (ex. estresse e susceptibilidade a doenças - Kindermann et al., 2012; Peterson et al., 2013; GCs, condição corpórea e fragmentação - Janin et al., 2011; Tennessen et al, 2014; sistema imune e GCs - Buehler et al., 2008; Dhabhar, 2014). Uma vez que o organismo coordena múltiplos sistemas fisiológicos para lidar com as demandas ambientais, incluindo a resposta a estressores, um estudo mais completo, integrando diversos aspectos fisiológicos se faz necessário para uma compreensão mais integrada destes sistemas. Os anfíbios são particularmente sensíveis a alterações ambientais, representando desta forma um grupo interessante como modelo de estudo para compreender as mudanças integradas de diversos aspectos fisiológicos, como GCs, hormônios reprodutivos, condição corpórea e sistema imune associados a resposta de estresse.

Dentro do presente contexto, desenvolver ferramentas apropriadas para a avaliação da resposta imune em animais não modelo, como os anuros, se faz necessário. Incialmente, a nossa pesquisa foi motivada pela necessidade de avaliar diferentes aspectos da resposta imune onde a aplicação possa ser amplamente utilizada em diversas espécies. Para tal, adaptamos protocolos de fagocitose a partir de macrófagos peritoneais, provenientes do uso em mamíferos, para o uso com sapos. A partir de metodologias para avaliação da resposta imune (capacidade bactericida do plasma e fagocitose de células peritoneais - desenvolvida durante este estudo), de dosagens hormonais no plasma e medidas de condição corpórea (amplamente utilizada na literatura), nós avaliamos o efeito da manutenção em cativeiro (comumente utilizado como agente estressor 
em vertebrados, incluindo anfíbios) sobre variáveis associadas à resposta de estresse e imunidade em sapos brasileiros. 


\section{CONCLUSÕES}

Em relação à utilização da citometria de fluxo como ferramenta para analises de fagocitose, podemos afirmar que a citometria convencional é um método confiável para estimar medidas de fagocitose. No entanto, a citometria de fluxo por imagem é um método mais preciso de análise no estudo da fagocitose, dispensando o uso de outras ferramentas associadas para as análises (sorting e imagem por microscopia convencional). Além disso, permite análises de parâmetros adicionais que não poderiam ser realizados pela citometria convencional, sendo estes também mais precisos (qualitativamente e quantitativamente) quando comparados à citometria convencional associada à a contagem manual (microscopia convencional).

O cativeiro por tempo prolongado pode ser considerado um agente estressor para Rhinella schneideri e $R$. icterica, uma vez que a manutenção prolongada dos sapos nestas condições $(R$. schneideri - 60 dias; $R$. icterica - 90 dias) resultou no aumento dos níveis plasmáticos de corticosterona, diminuição dos níveis plasmáticos de testosterona e da resposta imune em ambas as espécies.

A fagocitose de células peritoneais, tanto quando avaliada como porcentagem de fagocitose, quanto como eficiência de fagocitose, esteve positivamente correlacionada com os níveis plasmáticos de testosterona ao longo do tempo em cativeiro em ambas as espécies estudadas. Uma vez que esta relação se manteve independente da variação da condição corpórea, é possível que haja um papel imunomodulatório direto entre dos níveis plasmáticos de testosterona sobre a resposta imune celular em sapos.

Os níveis plasmáticos de corticosterona estiveram positivamente correlacionados com a capacidade bactericida plasmática quando não houve perda de condição corpórea em resposta ao cativeiro, indicando que a corticosterona pode exercer efeitos estimulatórios sobre a resposta imune quando o animal apresenta uma boa condição corpórea. Havendo variação da condição corpórea em condições de estresse crônico, a corticosterona plasmática pareceu influenciar 
indiretamente a capacidade bactericida plasmática através da influência sobre a condição corpórea do indivíduo, onde indivíduos com maior corticosterona plasmática apresentaram menor condição corpórea e menor capacidade bactericida plasmática ao longo do tempo em cativeiro. Nossos resultados sugerem que, a resposta imune inata parece estar associada às variações dos níveis plasmáticos de corticosterona e testosterona, assim como com à condição corpórea de anuros sob condições de estresse de manutenção a longo prazo em cativeiro. 


\section{REFERÊNCIAS}

Ahmed, S.A., Dauphinée, M.J., Talal, N. (1985). Effects of short-term administration of sex hormones on normal and autoinmune mice. J. Immunol. 134: 204-210.

Ahmed, S.A., Karpuzoglu, E., Khan, D. (2010). Effects of sex steroids on innate and adaptive immunity. In: Klein, S. L.; Roberts, C. W. (Eds), Sex hormones and immunity to infection. Germany: Springer Press. Pp. 27-51.

Assis, V. R. 2015. Níveis plasmáticos de corticosterona, testosterona e imunocompetência em Bufonídeos. Tese de doutorado, Universidade de São Paulo, São Paulo, SP, Brasil.

Assis, V.R., Titon, S.C.M., Barstotti, A.M.G., Titon Jr., B., Gomes, F.R. (2015). Effects of acute restraint stress, prolonged captivity stress and transdermal corticosterone application on immunocompetence and plasma levels of corticosterone on the cururu toad (Rhinella icterica). PlosOne 10(4): e0121005.

Assis, V.R., Titon, S.C.M., Queiroz-Hazarbassanov, N.G.T., Massoco, C.O., Gomes, F.R. (2017). Corticosterone transdermal application in toads (Rhinella icterica): effects on cellular and humoral immunity and steroid plasma levels. J. Exp. Zool. 327(4): 200-213.

Barriga, C., Martín, M.I., Tabla, R., Ortega, E., Rodríguez, A.B. (2001). Circadian rhythm of melatonin, corticosterone and phagocytosis: effect of stress. J. Pineal. Res. 30: 180-187.

Barriga, C., Martín, M.I., Ortega, E., Rodríguez, A.B. (2002). Physiological concentrations of melatonin and corticosterone in stress and their relationship with phagocytic activity. J. Neuroendocrinol. 14: 691-695.

Barsotti, A.M.G., Assis, V.R., Titon, S.C.M., Titon Jr., Ferreira, Z.F.S., Gomes, F.R. (2017). ACTH modulation on corticosterone, melatonin, testosterone and innate immune response in the treefrog Hypsiboas faber. Comp. Biochem. Physiol. A 204: 177-184.

Berger, S., Martin II, L.B., Wikelski, M., Romero, L.M., Kalko, E.K.V., Vitousek, M.N., Rödl, T. (2005). Corticosterone suppresses immune acytivity in territorial Galápagos marine iguanas during reproduction. Horm. Behav. 47: 419-429.

Bishop, C.R., Athens, J.W., Boggs, D.R., Warner, H.R., Cartwrig, G.E, Wintrobe, M.M. (1968). Leukokinetic Studies 13. A non-steady-state kinetic evaluation of mechanism of cortisone-induced granulocytosis. J. Clin. Investig. 47: 249-260.

Bourgeon, S., Raclot, T., Maho, Y.L., Ricquier, D., Criscuolo, F. (2007). Innate immunity, assessed by plasma NO measurements, is not suppressed during the incubation fast eiders. Dev. Comp. Immunol. 31: 720-728.

Bradley, A.J. (1987). Stress and mortality in the red-tailed phascogale, Phascogale calura (Marsupialia: Dasyuridae). Gen. Comp. Endocrinol. 67: 85-100.

Bradley, A.J., McDonald, L.R., Lee, A.K. (1980). Stress and mortality in a small marsupial (Antechinus stuartii, Macleay). Gen. Comp. Endocrinol. 40: 188-200.

Buehler, D.M., Bhola, N., Barjaktarow, D., Goymann, W., Schwabl, I., Tieleman, B.I., Piersma, T. (2008). Constitutive immune function responds more slowly to handling stress than corticosterone in a shorebird. Physiol. Biochem. Zool. 81(5): 673-681.

Casto, J.M., Nolan, V.Jr., Ketterson, E.D. (2001). Steroid hormones and immune function: experimental studies in wild and captive dark-eyed juncos (Junco hyemalis). Am. Nat. 157: 408-420. 
Cavalcanti, D.M.H., Lotufo, C.M.C., Borelli, P., Tavassi, A.M.C., Pereira, A.L.M., Markus, R.P., Farsky, S.H.P. (2006). Adrenal deficiency alters mechanisms of neutrophil mobilization. Mol. Cel. Endocrinol. 249: 32-39.

Chantong, B., Kratschmar, D.V., Nashev, L.G., Balazs, Z., Odermatt, A. (2012). Mineralocorticoid and glucocorticoid receptors differentially regulate NF-kappaB activity and pro-inflamatory cytokine production in murine BV-2 microglial cells. $\mathrm{J}$. Neuroinflamm. 9:260. Doi:10.1186/1742-2094-9-260

Chao, T.C., Van Alten, P.J., Walter, R.J. (1994). Steroid sex hormones and macrophage function: modulation of reactive oxygen intermediates and nitrite release. Am. J. Reprod. Immunol. 32(1): 43-52.

Ciliberti, M.G., Albenzio, M., Inghese, C., Santillo, A., Marino, R. Sevi, A., Caroprese, M. (2016). Peripheral blood mononuclear cell proliferation and cytokine production in sheep as affected by cortisol level and duration of stress. J. Dairy Sci. 100: 1-7.

Davis, A.K., Maney, D.L., Maerz, J.C. (2008). The use of leukocyte profiles to measure stress in vertebrates: a review for ecologists. Funct. Ecol. 22: 760-772.

Desprat, J.L., Lengagne, T., Dumet, A., Desouhant, E., Mondy, N. (2015). Immunocompetence handicap hypothesis in treefrog: trade-off between sexual signals and immunity? Behav. Ecol. 26(4): 1138-1146.

Deviche, P., Hurley, L., Fokidis, H., Lerbour, B., Silverin, B., Silverin, B., Sabo, J., Sharp, P.J. (2010). Acute stress rapidly decreases plasma testosterone in a free- ranging male songbird: potential site of action and mechanism. Gen. Comp. Endocrinol. 169: 82-90.

Dhabhar, F.S., McEwen, B.S. (1999). Enhancing versus suppessive effects of stress hormones on skin immune function. Proc. Natl. Acad. Sci. 96: 1059-1064.

Dhabhar, F.S. (2009). Enhancing versus suppressive effects of stress on immune function: implications for immunoprotection and immunopathology. Neuroimmunomodulat. 16: $300-317$.

Dhabhar, F.S. (2014). Effects of stress on immune function: the good, the bad, and the beautiful. Immunol. Res. 58: 193-210.

Evans, M.R., Goldsmith, A.R., Norris, S.R.A. (2000). The effects of testosterone on antibody production and plumage coloration in male house sparrows (Passer domesticus). Behav. Ecol. Sociobiol. 47: 156-163.

Gilliver, S.C. (2010). Sex steroids as inflammatory regulators. J. Steroid Biochem. 10: 105115.

Gomes, F.R., Oliveira, R.V., Assis, V.R., Titon Jr., B., Moretti, E.H., Mendonça, M.T. (2012). Interspecific variation in innate immune defenses and stress response of toads from Botucatu (São Paulo, Brazil). S. Am. J. Herpetol. 7(1): 1-8.

Grossman, C.J. (1984). Regulation of the immune system by sex steroids. Endocr. Rev. 5, 435455.

Grossman, C.J. (1985). Interactions between the gonadal steroids and the immune system. Science. 227: 257-261.

Graham, S.P., Kelehear, C., Brown, G.P., Shine, R. (2012). Corticosterone-immune interactions during captive stress in invading Australian cane toads (Rhinella marina). Horm. Behav. 62: $146-153$. 
Greenman, C.G., Martin, L.B., Hau, M. (2005). Reproductive state, but not testosterone, reduces immune function in male house sparrows (Passer domesticus). Physiol. Biochem. Zool. 78: 60-68.

Harms, N.J, Fairhurst, G.D., Bortolotti, G.R., Smits, J.E.G. (2010). Variation in immune function, body condition, and feather corticosterone in nestling tree swallows (Tachyneta bicolor) on reclaimed wetlands in the Athabasca oil sands, Alberta, Canada. Environ. Pollut. 158: 841-848.

Hoffman-Goetz, L., Keir, R., Thorne, R., Houston, M.E., Young, C. (1986). Chronic stress in mice depresses splenic T lymphocyte mitogenesis in vitro. Clin. Exp. Immunol. 66(3): 551-557.

Hou, J., Zheng, W.F. (1988). Effect of sex hormones on NK and ADCC activity of mice. Int. J. Immunopharm. 10: 15-22.

Inman, R.D. (1978). Immunologic sex differences and the female predominance in systemic lupus erythematosus. Arthritis Rheum. 21: 849-852.

Janin, A., Léna, J.P., Joly, P. (2011). Beyond occurrence: Body condition and stress hormone as integrative indicators of habitat availability and fragmentation in the common toad. Biol. Conserv. 144: 1008-1016.

Jones, S., Bell, K. (2004). Plasma corticosterone concentrations in males of the skink Egernia whitii during acute and chronic confinement, and over a diel period. Comp. Biochem. Physiol. Part A 137: 105-113.

Kindermann, C., Narayan, E.J., Hero, J. (2012). Urinary corticosterone metabolites and chytridiomycosis disease prevalence in a free-living population of male Stony Creek frogs (Litoria wilcoxii). Comp. Biochem. Physiol. Part A 162: 171-176.

Liao, J., Keiser, J.A., Scales, W.E., Kunkel, S.L., Kluger, M.J. (1995). Role of corticosterone in TNF and IL-6 production in isolated perfused rat liver. Am. J. Physiol. Reg I 268(3): R699-R706.

Lim, H-Y., Müller, N., Herold, M.J., Brandt, J van den., Reichardt, M. (2007). Glucocorticoids exert opposing effects on macrophage function dependent on their concentration. Immunol. 122: 47-53.

Marketon, J.I.W., Glaser, R. (2008). Stress hormones and immune function. Cel. Immunol. 252: $16-26$.

Martin, L.B., Weil, Z.M., Nelson, R.J. (2008). Seasonal changes in vertebrate immune activity: mediation by physiological trade-offs. Phil. Trans. R. Soc. B 363: 321-339.

McCormick, G.L., Langkilde, T. (2014). Immune responses of estern fence lizards (Sceloporus undulatus) to repeated acute elevation of corticosterone. Gen. Comp. Endocrinol. 204: 135-140.

McCrohon, J.A., Jessup, W., Handelsman, D.J., Celermajer, D.S. (1999). Androgen exposure increases human monocyte adhesion to vascular endothelium and endothelial cell expression of vascular cell adhesion molecule-1. Circulation 99(17): 2317-2322.

McDonald, I.R., Lee, A.K., Bradley, A.J., Than, K.A. (1981). Endocrine changes in dasyurid marsupiais with differing mortality patterns. Gen. Comp. Endocrinol. 11: 292-301.

McEwen, B.S., Biron, C.A., Brunson, K.W., Bulloch, K., Chambers, W.H. (1997). The role of adrenocorticoids as modulators of immune function in health and disease: Neural, endocrine and immune interactions. Brain Res. Rev. 23: 79-133. 
Mondal, S. Rai, U. (2002). In vitro of sex steroids on cytotoxic activity of splenic macrophages in wall lizard (Hemidactylus flaviviridis). Gen. Comp. Endocrinol. 125: 264-271.

Moore, M.C., Thompson, C.W., Marler, C.A. (1991). Reciprocal changes in corticosterone and testosterone levels following acute and chronic handling stress in the tree lizard, Urosaurus ornatus. Gen. Comp. Endocrinol. 81: 217-226.

Narayan, E.J., Hero, J., Cockrem, J.F. (2012). Inverse urinary corticosterone and testosterone metabolite responses to different durations of restraint in the cane toad (Rhinella marina). Gen. Comp. Endocrinol. 179: 345-349.

Nava-Castro, K., Hernández-Bello, R., Muñiz-Hernandez, S., Camacho-Arroyo, I., MoralesMontor, J. (2012). Sex steroids, immune system, and parasitic infections: facts and hypotheses. Na. N Y Acad. Sci. 1262: 16-26.

Neuman-Lee, L.A., Fokidis, H.B., Spence, A.R., Walt, M.V., Smith, G.D., Durham, S., French, S.S. (2015). Food restriction and chronich stress alter energy use and affects immunity in an infrequent feeder. Funct. Ecol. 29: 1453-1462.

Paolucci, M., Esposito, V., Di Fiore, M.M., Botte, V. (1990). Effects of short postcapture confinement on plasma reproductive hormone and corticosterone profiles in Rana esculenta during the sexual cycle. Bolletino di zoologia, 57: 253-259.

Peters, A. (2000). Testosterone treatment is immunosuppressive in superb fairy-wrens, yet freeliving males with high testosterone are more immunocompetent. Proc. R. Soc. Lond. B 267: 883-889.

Peterson, J.D., Steffen, J.E., Reinert, L.K., Cobine, P.A., Appel, A., Rollins-Smith, L., Mendonça, M.T. (2013). Host stress response is important for the pathogenesis of the deadly amphibian disease, Chytridiomycosis, in Litoria Caerulea. PLoS One, 8(4), e62146.

Riccardi, C., Bruscoli, S., Migliorati, G. (2002). Molecular mechanisms of immunomodulatory activity of glucocorticoids. Pharmacol. Res. 45(5): 361-368.

Roberts, M.L., Buchanan, K.L., Evans, M.R. (2004). Testing the immunocompetence handicap hypothesis: a review of the evidence. Anim. Behav. 68: 227-239.

Roberts, M.L., Buchanan, K.L., Hasselquist, D., Evans, M.R. (2007). Effects of testosterone and corticosterone on immunocompetence in the zebra finch. Horm. Behav. 51: 126-134.

Sapolsky, R.M., Romero, L.M., Munck, A.U. (2000). How do glucocorticoids influence stress responses? Integrating permissive, suppressive, stimulatory, and preparative actions. Endocr. Rev. 21:55-89.

Sapolsky, R.M. (2002). Endocrinology of the stress response. In: Becker, J.B., Reedlove, S.M., Crews, D., McCarthy, M.M. (eds.), Behavioral Endocrinology. Cambridge: MIT press. Pp. 409-450.

Savita, S., Rai, U. (1998). Sex steroid hormones modulate the activation of murine peritoneal macrophages: receptor mediated modulation. Comp. Biochem. Phusiol. 119(2): 199-204.

Tennessen, J.B., Parks, S.E., Langkilde, T. (2014). Traffic noise causes physiological stress and impairs breeding migration behavior in frogs. Conserv. Physiol. 2(1): cou032. DOI: 10.1093/conphys/cou032.

Thomas, J.R, Woodley, S.K. (2015). Treatment with corticosterone delays cutaneous wound healing in male and female salamanders. Gen. Comp. Endocrinol. 216: 33-38. 
Tokarz, R.R. Summers, C.H. (2011). Stress and reproduction in reptiles. In: Norris, D. O.; Lopez, K. H. eds. Hormones and reproduction in vertebrates. Vol. 3: reptiles. New York, NY: Academic Press. Pp. 169-213.

Tripathi, M.K., Singh, R. (2014). Differential suppressive effects of testosterone on immune function in fresh snake, Natrix piscator. An in vivo study. PlosOne 9(8): e104431.

Wiegers, G.J., Croiset G., Reul, J.M.H.M., Holsboer, F., De Kloet, E.R. (1993). Differential effects of corticosteroids on rat peripheral blood T-lymphocyte mitogenesis in vivo and in vitro. Am. J. Physiol. 265: E825-E830.

Wiegers, G.J., Labeur, M.S., Stec, I.E., Klinkert, W.E., Holsboer, F., Reul, J.M. (1995). Glucocorticoids accelerate anti-T cell receptor induced T cell growth. J. Immunol. 155: 1893-1902.

Wiegers, G.J., Reul, J.M.H.M. (1998). Induction of cytokine receptors by glucocorticoids; functional and pathological significance. Trends Pharmacol. Sci. 19: 317-321.

Yin, Z., Lam, T.J., Sim, Y.M. (1995). The effects of crowding stress on the non-specific immuneresponse in fancy carp (Cyprinus carpio L.). Fish Shellfish Immun. 5(7): 519529.

Zhong, H-J., Wang, H-Y., Yang, C., Zhou, J-Y., Jiang, J-X. (2013). Low concentrations of corticosterone exert stimulatory effects on macrophage function in a manner dependent on glucocorticoid receptors. Internat. J. Endocrinol. Doi: 10.1155/2013/405127. 